\title{
Evaluation of Crimean-Congo hemorrhagic fever suspected cases admitted to a secondary care hospital in Kastamonu, Turkey between 2014-2017
}

\author{
Hasan Tahsin Gozdas
}

Department of Infectious Diseases and Clinical Microbiology, Kastamonu State Hospital, Kastamonu,Turkey.

\begin{abstract}
Background: Crimean-Congo hemorrhagic fever (CCHF) is an endemic zoonosis in Kastamonu, Turkey. Clinical and laboratory findings may not be specific in the early phase of the disease, hence bringing a challenge to the clinician.

Objective: We aimed to distinguish CCHF cases among all suspected cases by comparing them with non-CCHF cases with respect to characteristics during admission.

Methods: Cases with a presumptive diagnosis of CCHF at a secondary care hospital in Kastamonu in between 2014-2017 were evaluated, retrospectively. CCHF and non-CCHF cases were compared with respect to their clinical, laboratory and epidemiological characteristics during admission.

Results: Among 76 suspected patients, CCHF was found in 46.1\% of them. Four-year fatality rate was $9.6 \%$ in CCHF cases. The frequency of headache, nausea/vomiting, leukopenia, thrombocytopenia less than 50x10\% $/ \mathrm{L}$, AST-ALT and LDH elevation, tick bites and contact with blood or body fluids of animals in CCHF cases were significantly higher than in non-CCHF cases $(\mathrm{p}<0.05)$.

Conclusion: Headache and nausea/vomiting accompanied with leukopenia, thrombocytopenia less than 50x10\% $/$ L, AST-ALT and LDH elevations raise the possibility of CCHF in endemic regions especially when there is a history of tick bite and contact with blood or body fluids of animals.

Keywords: Crimean-congo hemorrhagic fever, Kastamonu, Turkey.

DOI: https://dx.doi.org/10.4314/ahs.v19i1.16

Cite as: Gozdas HT. Evaluation of Crimean-Congo hemorrbagic fever suspected cases admitted to a secondary care hospital in Kastamonu, Turkey between 2014-2017. Afri Health Sci. 2019;19(1). 1433-1440. bttps:// dx.doi. org/10.4314/abs.v19i1.16
\end{abstract}

\section{Introduction}

Crimean-Congo hemorrhagic fever (CCHF) is a viral zoonotic infection caused by CCHF virus (CCHFV), a member of Bunyaviridae family. It is transmitted to humans through Hyalomma tick bites and blood or body fluids of infected animals or individuals. The clinical spectrum is wide from a self-limited disease to serious fatal infec-

\section{Corresponding author: \\ Hasan Tahsin Gozdas \\ Department of Infectious Diseases \\ and Clinical Microbiology, Kastamonu \\ State Hospital, Kastamonu,Turkey \\ Tel: +903662141053 \\ Fax: +903662142427 \\ Email: dr.htgozdas@yahoo.com.tr}

tion. The fatality rate was reported to be $10-40 \% 0^{1-7}$. The disease was reported in the Crimea in 1944 for the first time ${ }^{2}$. Thereafter, it has been reported from many regions of Africa, Asia, Eastern Europe, the Balkans and the Middle East ${ }^{6-9}$. In Turkey, the first CCHF case was seen in the Kelkit Valley region in 2002. CCHF is an endemic zoonosis in Turkey occurring every year in spring and summer months. The number of annually reported cases in Turkey is nearly $1000^{1-7}$. Compared with the countries that report $\mathrm{CCHF}$ cases, the highest number of $\mathrm{CCHF}$ cases has been reported annually from Turkey. Most of the cases in Turkey were from 15 cities in Kelkit Valley region and its environs including Kastamonu Province ${ }^{4,9-14}$. The fatality rate of CCHF in Turkey changes between 3\% and $9 \% 0^{15-17}$.

There are a limited number of studies investigating char- 
acteristics of patients with a presumptive diagnosis of CCHF during admission ${ }^{1,16-20}$. In this study, we aimed to reveal characteristics of $\mathrm{CCHF}$ suspected cases admitted to a secondary care hospital in Kastamonu, Turkey between 2014-2017. It was also investigated whether there is a difference between CCHF cases and non-CCHF cases in terms of clinical, laboratory and epidemiological characteristics.

\section{Methods}

Patients with a presumptive diagnosis of CCHF admitted to inpatient clinic in Kastamonu State Hospital (Kastamonu Province, Turkey) between 2014-2017 were enrolled in this study. A presumptive diagnosis of CCHF was made if cases met minimum two of the five following criteria: sudden onset high fever, headache, weakness, nausea/vomiting and diarrhea plus one of the following two criteria: thrombocytopenia (platelet count of $<150$ $\mathrm{x} 10^{9} / \mathrm{L}$ ), leukopenia (white blood cell count of $<4 \mathrm{x}$ $10^{9} / \mathrm{L}$ ), and also one of the below criteria: history of tick bite, close contact with animals, living in or travelling to rural areas, contact with people with similar symptoms in the past two weeks.

Serum samples of the cases were obtained upon admission and were sent to the Public Health Institution of Turkey, National Virology Reference Laboratory to investigate CCHF IgM antibodies by ELISA and CCHFV RNA by PCR. ELISA was not performed on serum samples if positive viral RNA was detected by PCR.

When CCHFV RNA or CCHF IgM was detected positive, CCHF diagnosis was confirmed. If both viral RNA and IgM antibody were negative, CCHF diagnosis was excluded. Cases with a history of suspected CCHF in the past four months were excluded from the study because serum CCHF IgM antibodies detected by ELISA can remain positive during four months $s^{2,4,6,7}$. The incubation period was defined as the interval between tick contact and appearance of symptoms. Cases diagnosed with hantavirus infection were excluded from the study, since hantavirus infection and $\mathrm{CCHF}$ are both viral hemorrhagic fevers that have similar clinical and laboratory properties. CCHF cases were evaluated retrospectively with respect to their clinical and laboratory values during admission to the hospital and epidemiological data within two weeks, and these characteristics of $\mathrm{CCHF}$ cases were compared with non-CCHF cases.

Statistical analysis was performed using SPSS version 15.0 (SPSS Inc., USA). Descriptive statistics (median, minimum and maximum value, mean and standard deviation, count and percentage) were used to summarize the results. Nominal variables were compared with $\chi^{2}$ test and Fisher's exact test. Continuous variables were compared with Student's t test and Mann Whitney U test. A p value of less than 0.05 was accepted as statistically significant.

\section{Results}

In total, 79 cases with suspected CCHF were followed up as inpatients in our hospital between 2014-2017. Three cases with hantavirus infection were excluded from the study. So, the remaining 76 cases were subjected to our study.

Two had a positive IgM by ELISA and 29 cases had a positive PCR for CCHFV RNA. So, a total of 31 cases $(40.7 \%)$ were diagnosed with definitive CCHF. The remaining 45 cases $(59.3 \%$ ) were found negative by both tests, also they did not get a specific infectious disease diagnosis. So, they were considered as non-CCHF cases. $61.3 \%$ (19) of the CCHF cases were female, while $38.7 \%$ (12) were male. However, $48.9 \%$ (22) and 51.1\% (23) of the non-CCHF cases were female and male, respectively. The mean age of CCHF cases and non-CCHF cases was $52.8 \pm 16.1$ and $54.4 \pm 19.1$, respectively. There were no statistically significant differences between the two groups regarding gender and age ( $p>0.05) .20$ cases from the CCHF group were referred to a tertiary care hospital, because their thrombocyte levels decreased below $50 \times 10^{9} / \mathrm{L}$ during follow-up. Three of the referred CCHF cases deceased while the other 17 cases survived. Cases who deceased were all female and they were 39,66 and 79 years old. They had no underlying diseases and mortality was due to multi-organ failure. Hence, the fatality rate was $9.6 \%(3 / 31)$ in the CCHF group.

Monthly distribution of the CCHF and non-CCHF cases was shown in Figure 1. It was seen that the earliest case was detected in February and the highest number of cases was admitted in June. Annual distribution of CCHF and non-CCHF cases was shown in Figure 2. It was seen that the number $\mathrm{CCHF}$ suspected admissions and confirmed cases had gradually decreased from 2014 to 2017. 


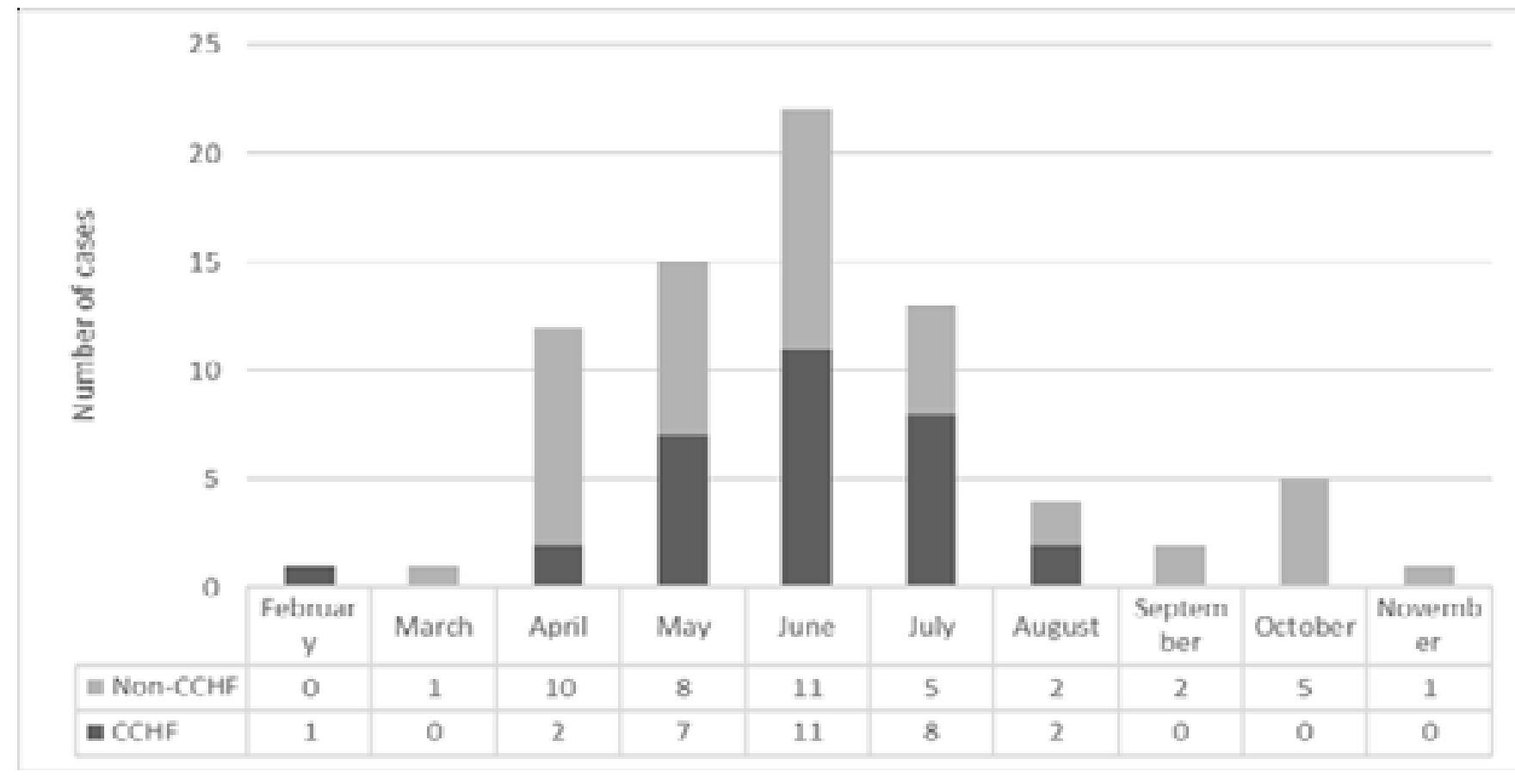

Figure 1: Monthly distribution of $\mathrm{CCHF}$ and non-CCHF cases during a four-year period.

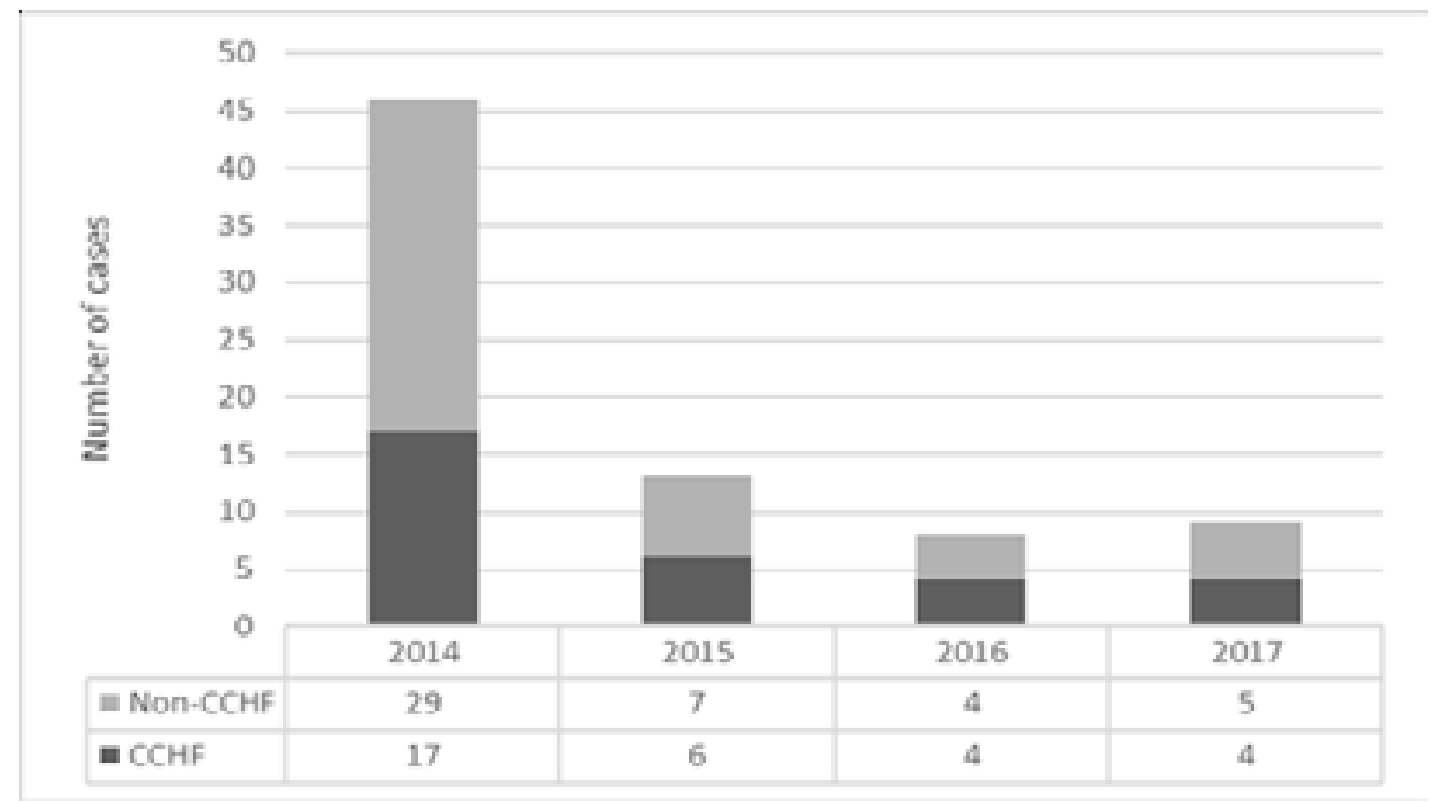

Figure 2: Annual distribution of CCHF and non-CCHF cases between 2014-2017.

Clinical symptoms of the cases during admission were summarized in Table 1. The most common physical finding during admission was fever, which was present in $61.3 \%$ (19) of $\mathrm{CCHF}$ and $66.7 \%$ (30) of non-CCHF cases. In the CCHF group, three $(9.7 \%)$ cases had maculopapular rash, one $(3.2 \%)$ had altered level of conscious- ness, hypotension and tachycardia and another one (3.2\%) had petechiae and hypotension during admission. In the non-CCHF group, three $(6.7 \%)$ cases had hemorrhagic findings, three $(6.7 \%)$ had hypotension and tachycardia, two $(4.4 \%)$ had maculopapular rash, and one $(2.2 \%)$ had ecchymosis and altered level of consciousness. There 
was no statistically significant difference between the two groups in terms of physical findings during admission $(p>0.05)$. Laboratory findings of the cases during admission are summarized in Table 2.

Table 1: Clinical symptoms of the cases with a presumptive diagnosis of Crimean-Congo hemorrhagic fever during admission

\begin{tabular}{|c|c|c|c|}
\hline & $\begin{array}{l}\text { CCHF cases } \\
\text { (31) }\end{array}$ & Non-CCHF cases(45) & p value \\
\hline Clinical symptoms & $\%(n)$ & $\%(n)$ & \\
\hline Fatigue & $100(31)$ & $100(45)$ & $\mathrm{p}>0.05$ \\
\hline Widespread myalgia & $100(31)$ & $91.1(41)$ & $\mathrm{p}>0.05 *$ \\
\hline Fever & $96.8(30)$ & $82.2(37)$ & $\mathrm{p}>0.05^{*}$ \\
\hline Headache & $96.8(30)$ & $75.6(34)$ & $\mathbf{p}<\mathbf{0 . 0 5 *}$ \\
\hline Nausea and vomiting & $83.9(26)$ & $51.1(23)$ & $\mathbf{p}<\mathbf{0 . 0 5}$ \\
\hline Abdominal pain & $41.9(13)$ & $28.9(13)$ & $\mathrm{p}>0.05$ \\
\hline Diarrhea & $38.7(12)$ & $20(9)$ & $\mathrm{p}>0.05$ \\
\hline Maculopapular rash & $9.7(3)$ & $4.4(2)$ & $\mathrm{p}>0.05^{*}$ \\
\hline Altered consciousness & $3.2(1)$ & $13.3(6)$ & $\mathrm{p}>0.05^{*}$ \\
\hline Bleeding & $9.7(3)$ & $6.7(3)$ & $\mathrm{p}>0.05 *$ \\
\hline
\end{tabular}

* Fisher's exact test

Table 2: Laboratory findings of cases with a presumptive diagnosis of Crimean-Congo hemorrhagic fever during admission

\begin{tabular}{llll}
\hline & CCHF cases (31) & $\begin{array}{l}\text { Non-CCHF } \\
\text { cases(45) }\end{array}$ & p value \\
\hline $\begin{array}{l}\text { Laboratory findings } \\
\text { Thrombocytopenia }\end{array}$ & \% (n) & \% (n) & \\
Platelet count: & & $77.8(35)$ & $\mathrm{p}>0.05^{*}$ \\
$\mathbf{1 0 1 - 1 5 0 \times 1 0} / \mathbf{L}$ & $32.3(10)$ & $31.1(24)$ & $\mathrm{p}>0.05$ \\
$\mathbf{5 1 - 1 0 0 \times 1 0} / \mathbf{L}$ & $25.8(8)$ & $46.6(21)$ & $\mathrm{p}>0.05$ \\
$\mathbf{< 5 0 \times 1 0} / \mathbf{L}$ & $35.5(11)$ & $2.2(1)$ & $\mathbf{p}<\mathbf{0 . 0 5 *}$ \\
Leukopenia & $93.5(29)$ & $57.8(26)$ & $\mathbf{p}<\mathbf{0 . 0 5}$ \\
AST-ALT elevation & $67.7(21)$ & $42.2(19)$ & $\mathbf{p}<\mathbf{0 . 0 5}$ \\
LDH elevation & $87.1(27)$ & $55.6(25)$ & $\mathbf{p}<\mathbf{0 . 0 5}$ \\
CK elevation & $51.6(16)$ & $31.1(14)$ & $\mathrm{p}>0.05$ \\
Anemia & $9.7(3)$ & $15.6(7)$ & $\mathrm{p}>0.05^{*}$ \\
INR elevation & $32.3(10)$ & $15.6(7)$ & $\mathrm{p}>0.05$ \\
\hline$*$ Fish's & &
\end{tabular}

When the cases were evaluated according to their occupations, most of them were farming and animal husbandry with $66.7 \%$ (16) and $71 \%$ (22) in the CCHF and the nonCCHF groups, respectively. There was no occupational risk in the remaining cases. Epidemiological data of the cases within the last two weeks were summarized in Table
3. The mean incubation period of cases with tick exposure was $3.8 \pm 2.7$ days in the CCHF group. The median day of illness on which patients were admitted was $2.0(0-$ 6) and 1.0 (0-7) days in CCHF and non-CCHF patients, respectively and the difference was not statistically significant ( $>>0.05)$. 
Table 3: Epidemiological data of cases with a presumptive diagnosis of Crimean-Congo hemorrhagic fever in the last two weeks

\begin{tabular}{|c|c|c|c|c|}
\hline & $\begin{array}{l}\text { CCHF } \\
\text { (31) }\end{array}$ & cases & $\begin{array}{l}\text { Non-CCHF } \\
\operatorname{cases}(45)\end{array}$ & p value \\
\hline Epidemiological data & $\%(n)$ & & $\%(n)$ & \\
\hline Living in rural area & $100(31)$ & & $91.1(41)$ & $\mathrm{p}>0.05^{*}$ \\
\hline Animal contact & $71(22)$ & & $53.4(24)$ & $\mathrm{p}>0.05$ \\
\hline Tick bite & $74.2(23)$ & & $44.4(20)$ & $\mathbf{p}<\mathbf{0 . 0 5}$ \\
\hline $\begin{array}{l}\text { Contact with blood or } \\
\text { body fluids of animals }\end{array}$ & $54.8(17)$ & & $28.9(13)$ & $\mathbf{p}<\mathbf{0 . 0 5}$ \\
\hline Travelling to rural area & $22.6(7)$ & & $11.1(5)$ & $\mathrm{p}>0.05^{*}$ \\
\hline $\begin{array}{l}\text { Contact with CCHF } \\
\text { cases }\end{array}$ & $0(0)$ & & $4.4(2)$ & $\mathrm{p}>0.05^{*}$ \\
\hline
\end{tabular}

* Fisher's exact test

\section{Discussion}

In this study, we found that the frequency of headache, nausea/vomiting, leukopenia, thrombocytopenia less than $50 \times 10^{\circ} / \mathrm{L}$, AST-ALT and LDH elevation, tick bites and contact with blood or body fluids of animals in $\mathrm{CCHF}$ cases were significantly higher than non-CCHF cases.

CCHFV may be transmitted through the bite of an infected tick or by exposure to blood or body fluids of a viremic animal or human ${ }^{23,6,7}$. Tick bites and animal husbandry are well-known risk factors for seropositivity. Accordingly, history of tick bite and animal husbandry was identified in most CCHF cases in Turkey. Seroprevalence was reported as $10 \%$ in persons with a history of tick bite. However, seroprevalence was reported higher in endemic regions of Turkey (10-19.6\%). Seropositivity in humans with a history of farming, animal husbandry, contact with animals and contact with ticks was reported quite high: $80 \%, 70 \%, 76 \%$ and $70 \%$, respectively ${ }^{4,5,21-25}$. Contact with animals was more commonly reported in CCHF cases than non-CCHF cases, but, a history of tick bite was similar between the two groups in Bolu, Turkey ${ }^{17}$. Similar to a previous study conducted in our hospital ${ }^{19}$, we found that the frequency of tick bites was significantly higher in CCHF cases than non-CCHF cases $(p<0.05)$. Other endemic tick-borne diseases in Kastamonu are tularemia and rickettsiosis, however their clinical presentations are greatly different from CCHF.

CCHF cases are frequently seen between April and September, with a peak incidence in June and July ${ }^{2,3,6,7}$. The majority of CCHF cases occurs during May, June and July $^{4}$. Consistently, most CCHF cases in our study was seen in June.

The number of male and female CCHF cases was found similar in Turkey. Individuals living in rural area and working in farming and animal husbandry are under risk for CCHF. Consistently, most CCHF cases are farmers and animal husbanders. Housewives in rural areas participate actively in farming and animal husbandry in Turkey $^{19,21-28}$. No housewives were present in our study, since housewives generally live in rural areas and actively work in farming or animal husbandry in Kastamonu, Turkey. ${ }^{19}$

In Turkey, the proportion of health care staff with CCHF among the whole cases between 2004-2007 was found $0.4 \%$ and seroprevalence among healthcare staff in endemic areas was found $2 \%$. In our study, there was no healthcare staff diagnosed with CCHF. The proportion of CCHF cases among all suspected cases was found $40.7 \%$ in our study. No statistically significant difference was found between the two groups in terms of gender $(p>0.05)$.

The most widespread symptoms in CCHF cases are fever, headache, myalgia, nausea, vomiting, abdominal pain, diarrhea and bleeding, respectively ${ }^{4,6,7}$. In some previous studies, fever, bleeding, vomiting, headache and diarrhea were reported more frequently in CCHF cases than non-CCHF cases ${ }^{17,18}$. In our study, headache and nausea/vomiting were reported more frequently in $\mathrm{CCHF}$ 
cases compared with non-CCHF cases $(p<0.05)$. There were no significant differences between the two groups in terms of clinical findings during admission ( $p>0.05)$. In suspected cases, headache and nausea/vomiting may help to differentiate CCHF cases from non-CCHF cases. The number of patients with hemorrhagic findings among CCHF and non-CCHF patients during admission was three in each group and the frequency was $9.7 \%$ and $6.7 \%$, respectively. Contrary to expectations, there is no statistically significant difference in terms of hemorrhagic findings between the groups ( $p>0.05)$. This may be because patients were admitted in the initial period of CCHF. In our study, the median day of illness on which CCHF cases applied to hospital was 2.0 days. Except our hospital, there is another private hospital in Kastamonu province that serves CCHF suspected cases. Cases with hemorrhagic findings might have been admitted to other hospitals/centers/departments and perhaps they were misdiagnosed in other medical departments.

Leukopenia, thrombocytopenia, raised AST-ALT, CK and LDH levels are the most widespread laboratory abnormalities in CCHF cases ${ }^{1,4,6,7}$. In some previous studies, thrombocytopenia, elevated AST-ALT and LDH levels were reported more frequently among CCHF cases than non-CCHF cases $^{17,18}$. In our study; leukopenia, AST-ALT and $\mathrm{LDH}$ elevations were more frequently seen in $\mathrm{CCHF}$ cases $(p<0.05)$, but, other laboratory findings during admission were found similar between the two groups ( $p>0.05)$. Thrombocytopenia independent from quantitative value was not statistically different between the two groups ( $p>0.05)$, however thrombocyte count less than $50 \times 10^{9} / \mathrm{L}$ was more frequently seen in CCHF cases $(p<0.05)$. So, leukopenia, thrombocytopenia less than $\left.50 \times 10^{9} / \mathrm{L}\right)$, AST-ALT and LDH elevations may help to distinguish $\mathrm{CCHF}$ cases from non-CCHF cases.

The fatality rate of $\mathrm{CCHF}$ is about $10-40 \%{ }^{1}$. The mean fatality rate for Turkey was nearly $5 \%$ between 2002 2007. The fatality rate was determined as $8.8 \%$ between 2006-2012 in Bolu Province, $250 \mathrm{~km}$ distant in the West of Kastamonu Province in our country ${ }^{17}$. In our hospital, 342 CCHF cases were diagnosed between 2005-2010 and the fatality rate was reported to be $2.9 \%{ }^{15}$. However, 19 CCHF cases were diagnosed in our hospital in 2013 and the fatality rate was determined as $5.3 \%{ }^{19}$. In our study, 31 cases were diagnosed with CCHF in 2014-2017 and the fatality rate was $9.6 \%$. This fatality rate was consistent with the classical literature and similar to the aforementioned study conducted in Bolu, but higher than other studies conducted in Turkey. This may be due to the fact that milder cases were less diagnosed, since most outpatients were not tested for CCHF unless their clinical and laboratory status exacerbated. Another reason may be that more severe patients were admitted to our hospital compared to the other hospital in Kastamonu. The fatality differences among studies in different regions may be associated with the evolution of the virus due to the climate changes in the course of time. The number of patients admitted with a presumptive diagnosis of CCHF in 2014-2017 had gradually decreased. This may be explained by the climate changes and changes in the route of migratory birds ${ }^{29,30}$.

The efficacy of ribavirin in CCHF treatment is still controversial. Although many papers advocated a therapeutic benefit related to ribavirin use, the quality of the evidence is low. CCHF treatment is generally based on supportive treatment ${ }^{6,7,31,32}$. In our study, all cases were managed with supportive treatment, none of them received ribavirin treatment.

Before any conclusion, we should declare study limitations. First, this was a retrospectively single center study with a small number of patients. Second, the study evaluates characteristics of patients during admission, the patients could not be evaluated during the course of the disease until the patient outcome. Despite these limitations, all patients admitted to our inpatient clinic with CCHFlike presentation during 2014-2017 were subjected to this study. The number of studies evaluating cases with a presumptive diagnosis of CCHF is rather limited ${ }^{1,16-20}$. Thus, more comprehensive studies including large number of patients are required.

CCHF still saves its significance in Kastamonu region of Turkey. According to studies from Turkey, fatality rate ranges between $5 \%$ and $10 \%$. Although the number of annual suspected cases in Kastamonu tended to decrease between 2014-2017, the fatality rate in a four-year period was found $9.6 \%$ in this study similar to other endemic countries $^{1-7,15-17}$. At first visit, clinical presentation, physical and laboratory findings may not be specific in CCHF especially in the initial period of the disease $e^{2,3,5-7}$. In endemic regions, clinicians should consider the possibility 
of other diseases in cases with CCHF-like presentation and distinguish the clinical, laboratory and epidemiological features of CCHF cases during admission.

\section{Conclusion}

According to the results of our study, clinical symptoms like headache and nausea/vomiting accompanied with the laboratory findings like leukopenia, thrombocyte count less than $50 \times 10^{9} / \mathrm{L}$, AST-ALT and LDH elevations raise the possibility of CCHF in endemic regions especially when there is a history of tick bite and contact with blood or body fluids of animals. Clinicians should be more careful in the management of such cases in order to prevent undesired outcomes.

\section{Source of support}

Nil.

\section{Conflict of interest}

None to declare.

\section{References}

1. Kilinc C, Gückan R, Capraz M, et al. Examination of the specific clinical symptoms and laboratory findings of Crimean-Congo hemorrhagic fever. J Vector Borne Dis. $2016 ; 53: 162-7$.

2. Whitehouse CA. Crimean-Congo haemorrhagic fever. Antiviral Res. 2004; 64: 145-60.

3. Ergönül O. Crimean-Congo haemorrhagic fever. Lancet Infect Dis. 2006; 6: 203-14.

4. Yilmaz GR, Buzgan T, Irmak H, et al. The epidemiology of Crimean-Congo hemorrhagic fever in Turkey, 2002-2007. Int J Infect Dis. 2009; 13: 380-6.

5. Bodur H, Akinci E, Ascioglu S, Öngürü P, Uyar Y. Subclinical infections with Crimean-Congo hemorrhagic fever virus, Turkey. Emerg Infect Dis. 2012; 18: 640-2.

6. Bente DA, Forrester NL, Watts DM, McAuley AJ, Whitehouse CA, Bray M. Crimean-Congo hemorrhagic fever: history, epidemiology, pathogenesis, clinical syndrome and genetic diversity. Antiviral Res. 2013; 100: 15989.

7. Mertens M, Schmidt K, Ozkul A, Groschup MH. The impact of Crimean-Congo hemorrhagic fever virus on public health. Antiviral Res. 2013; 98: 248-60.

8. Sidira P, Maltezou HC, Haidich AB, Papa A. Seroepidemiological study of Crimean-Congo haemorrhagic fever in Greece, 2009-2010. Clin Microbiol Infect. 2012; 18: E169.

9. Vorou RM, Papavassiliou VG, Tsiodras S. Emerging zoonoses and vector-borne infections affecting humans in Europe. Epidemiol Infect. 2007; 135: 1231-47.

10. Karti SS, Odabasi Z, Korten V, et al. Crimean-Congo hemorrhagic fever in Turkey. Emerg Infect Dis. 2004; 10 : 1379-84.

11. Günaydın NS, Aydın K, Yılmaz G, Çaylan R, Köksal I. Crimean-Congo hemorrhagic fever cases in the Eastern Black Sea Region of Turkey: demographic, geographic, climatic, and clinical characteristics. Turk JMed Sci. 2010; 40: 829-34.

12. Chinikar S, Ghiasi SM, Hewson R, Moradi M, Haeri A. Crimean-Congo hemorrhagic fever in Iran and neighboring countries. J Clin Virol. 2010; 47: 110-4.

13. Gergova I, Kunchev M, Kamarinchev B. Crimean-Congo haemorrhagic fever virustick survey in endemic areas in Bulgaria. J Med Virol. 2012; 84: 608-14.

14. Ergonul O. Crimean-Congo haemorrhagic fever virus: new outbreaks, new discoveries. Curr Opin Virol. 2012; 2: 215-20.

15. Ozbey SB, Kader Ç, Erbay A, Ergönül Ö. Early use of ribavirin is beneficial in Crimean-Congo hemorrhagic fever. Vector Borne Zoonotic Dis. 2014; 14:300-2.

16. Erenler AK, Kulaksiz F, Ülger H, et al. Characteristics of patients admitted to the emergency department due to tick bite. Trop Doct. 2014; 44: 86-8.

17. Duran A, Küçükbayrak A, Ocak T, et al. Evaluation of patients with Crimean- Congo hemorrhagic fever in Bolu, Turkey. Afr Health Sci. 2013; 13: 233-42 PubMed .

18. Mostafavi E, Pourhossein B, Chinikar S. Clinical symptoms and laboratory findings supporting early diagnosis of Crimean-Congo hemorrhagic fever in Iran. $J$ Med Virol. 2014; 86: 1188-92.

19. Hekimoğlu HC, Demirci NA. Evaluation of cases with a preliminary diagnosis of Crimean- Congo hemorrhagic fever and comparison of characteristics in patients admitted to a secondary care hospital in Kastamonu, Turkey. Afr Health Sci. 2014 ;14:873-81.

20. Kuchuloria T, Imnadze P, Chokheli M, et al. Viral Hemorrhagic Fever Cases in the Country of Georgia: Acute Febrile Illness Surveillance Study Results. Am JTrop Med Hyg. 2014. pii: 13-0460.

21. Yagci-Caglayik D, Korukluoglu G, Uyar Y. Seroprevalence and risk factors of Crimean-Congo hemorrhagic 
fever in selected seven provinces in Turkey. J Med Virol. 2014; 86: 306-14.

22. Gunes T, Engin A, Poyraz O, et al. Crimean-Congo hemorrhagic fever virusin high-risk population, Turkey. Emerg Infect Dis. 2009;15: 461-4.

23. Gargili A, Midilli K, Ergonul O, et al. Crimean-Congo hemorrhagic fever in European part of Turkey: Genetic analysis of the virus strains from ticks and a seroepidemiological study in humans. Vector Borne Zoonotic Dis. 2011; 11: 747-52.

24. Ertugrul B, Kirdar S, Ersoy OS, et al. The seroprevalence of Crimean-Congo haemorrhagic fever among inhabitants living in the endemic regions of Western Anatolia. Scand J Infect Dis. 2012; 44: 276-81.

25. Koksal I, Yilmaz G, Aksoy F, Erensoy S, Aydin H. The seroprevalance of Crimean-Congo haemorrhagic fever in people living in the same environment with Crimean-Congo haemorrhagic fever patients in an endemic region in Turkey. Epidemiol Infect. 2014; 142: 239-45.

26. Tekin S, Barut S, Bursali A, et al. Seroprevalence of Crimean-Congo haemorrhagic fever $(\mathrm{CCHF})$ in risk groups in Tokat Province of Turkey. Afr J Microbiol Res. 2010; 4: 214-7.
27. Cevik MA, Uzun R, Yilmaz N, et al. Risk factors for Crimean-Congo haemorrhagic fever outbreak in Central Anatolia: a case control study. R2227. 15 ${ }^{\text {th }}$ European Congress of Clinical Microbiology and Infectious Diseases (ECCMID). 2005.

28. Ozkurt Z, Kiki I, Erol S. Crimean-Congo hemorrhagic fever in Eastern Turkey: clinical features, risk factors and efficacy of ribavirin therapy. J Infect 2006;52: 207-15. 29. Aker S, Akıncı H, Kılıçoğlu C, Leblebicioglu H. The Geographic Distribution of Cases of Crimean-Congo hemorrhagic fever: Kastamonu, Turkey. Ticks Tick Borne Dis. 2015;6:730-6.

30. Leblebicioglu H, Eroglu C, Erciyas-Yavuz K, Hokelek M, Acici M, Yilmaz H. Role of migratory birds in spreading Crimean-Congo hemorrhagic fever, Turkey. Emerg Infect Dis. 2014;20:1331-4.

31. Soares-Weiser K, Thomas S, Thomson G, Garner P. Ribavirin for Crimean- Congo hemorrhagic fever: systematic review and meta-analysis. BMC Infect Dis. 2010; 10: 207.

32. Salehi M. Evaluation of the efficacy of ribavirin therapy on survival of Crimean- Congo hemorrhagic fever patients: a case-control study. Jpn J Infect Dis. 2009;62:11-15. 